\title{
Optimization of Oil Pads on a Hydrostatic Turntable for Supporting Energy Conservation Based on Particle Swarm Optimization
}

\author{
Yumo Wang ${ }^{1}$ - Zhifeng Liu ${ }^{1}{ }^{*}$ - Ligang Cai ${ }^{1}$ - Qiang Cheng1 - Xiangmin Dong2 \\ ${ }^{1}$ Beijing University of Technology, Key Laboratory of Advanced Manufacturing Technology, China \\ 2 Chengde Petroleum College, Department of Mechanical Engineering, China
}

The performance of hydrostatic turntables is strongly influenced by the geometry of oil pads. The properly designed structure of oil pads can reduce the power consumption of hydrostatic turntables and improves their service performance. A structural optimization of oil pads on a hydrostatic turntable based on particle swarm optimization is presented to improve the carrying performance and decrease energy costs. Using the finite difference method, the Reynolds equation is resolved to calculate the pressure distribution and flow rate in oil pads. The power cost of oil supply is determined according to the turntable working condition, and minimal energy consumption is set as the optimal solution. The geometrical parameters of the oil pad are chosen as individual elements that will converge to an optimal result by using PSO.

Keywords: hydrostatic turntable, Reynolds equation, PSO, energy conservation

\begin{abstract}
Highlights
- $\quad$ Performance characteristics, such as pressure distribution and oil flow rate, are determined by the Reynolds equation and finite difference method (FDM).

- The relationship between oil pocket scale and supporting power consumption is determined numerically. Reliability of the algorithm is verified by conducting experiments on a miniature testing table.

- $\quad$ PSO is carried out to solve the optimal pocket size for minim pump power. An efficient and practical optimization method for hydrostatic turntable design is presented.
\end{abstract}

\section{INTRODUCTION}

The hydrostatic turntable is a key part of heavy-duty computerized numerical control (CNC) machine tools, which plays a supporting role and provides high precision rotary motion. The external supply system continuously pumps pressurized oil into oil pads. The cost of running a hydrostatic turntable can be reduced by reducing the power consumption of oil pads. The analysis and optimization of hydrostatic bearing are complex, requiring many numerical computations. The geometrical size of oil pad is accurate to millimetres, considering processing feasibility. Therefore, an optimization algorithm with high efficiency and acceptable accuracy is needed to optimize hydrostatic bearing; particle swarm optimization (PSO) is an efficient computational method, suitable for efficiently reducing computational load.

Wang et al. [1] presented a fast-convergent numerical method to solve the modified Reynolds equation. Singh et al. [2] applied different types of modified Reynolds equations in analysing different shapes of oil pads. Liu et al. [3] provided the general type of the Reynolds equation of circular and rectangular oil pads. The variation of viscosity caused by temperature changes was ignored in the study of El Khlifi et al. [4] based on the low moving speed working condition. Mankovits et al. [5] conducted his optimal research by using a support vector regression method. Gustafsson et al. [6] introduced changing viscosity in the Reynolds equation. Yang et al. [7] introduced non-Newtonian characteristics into the Reynolds equation. Masjedi and Khonsari [8] solved the Reynolds equation by the efficient numerical method of successive over-relaxation (SOR). Getachew and Prawal [9] analysed the lubrication performance of a slider by using finite different method (FDM). Cai et al. [10] optimized the oil pad structure to improve the stiffness of the hydrostatic guideway. Weißbacher et al. [11] reformed the film clearance to improve the support performance of hydrostatic journal bearing. Cheng et al. [12] applied PSO to diverse types of hydrostatic and aerostatic bearing. Chang and Jeng [13] designed a double-pad aerostatic bearing using PSO. Chan [14] conducted an optimization research on hybrid journal bearings by PSO. Soltani-Mohammadi et al. [15] proved that the PSO is more efficient in computing in comparison to a simulated annealing method. Zheng and Liao [16] used social emotional PSO (SEPSO). Srisha Rao and Jagadeesh [17] improved the convergence of an optimization algorithm by using vector evaluated PSO (VEPSO). Flocker and Bravo [18] discussed the global convergence of PSO in their article. Tang et al. [19] 
applied PSO in the energy conservation of a workshop. Luo et al. [20] proposed a dynamic optimal solution based on Species-based PSO (SPSO). Liu et al. [21] made a comparison between different optimization methods in a review. All the above studies show that PSO is a broadly applicable optimization method for many circumstances.

A practical optimization method based on PSO for a hydrostatic turntable is proposed in this work to improve its service performance. The Reynolds equation of the hydrostatic turntable is modified according to the working condition. Performance characteristics, such as pressure distribution and oil flow rate, are determined by FDM. The geometry size of oil pads is selected as individuals in the optimization progress. With minimum film thickness set to a constant safety level, the power consumption is chosen as the fitness of each individual. Finally, through the PSO algorithm, the optimal solution is determined.

\section{HYDROSTATIC TURNTABLE MODELING AND GENERAL EQUATIONS}

\subsection{Model of Hydrostatic Turntable}

A hydrostatic turntable is an essential part of heavy machine tools, which is supported by several oil pads on the base. A model of a hydrostatic turntable is shown in Fig. 1.

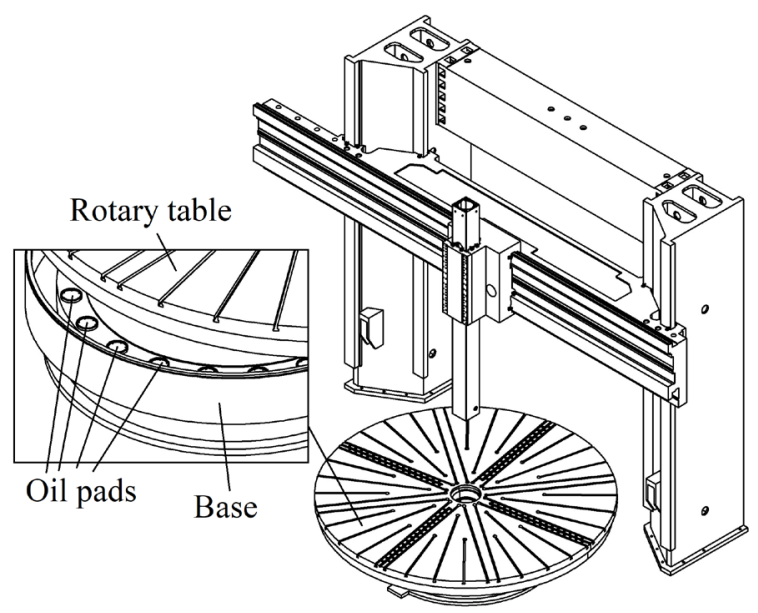

Fig. 1. Model of hydrostatic turntable on heavy machine tools

The supporting oil pad of hydrostatic turntable is a circular step pad with concentric oil pockets, and there are $N$ supporting pads on the rotary table. A model of a circular oil pad is shown in Fig. 2, where $R_{0}$ is the radius of oil pad, $R_{1}$ is the radius of oil pocket, $H$ is the oil film thickness of the supporting pad.
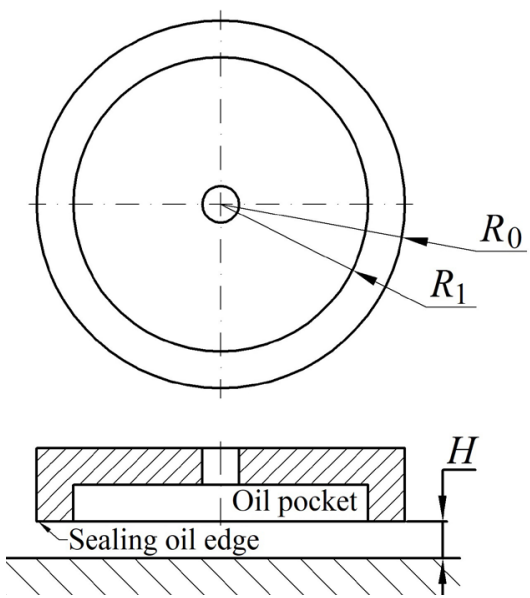

Fig. 2. The structure of the supporting oil pad

In [10], the load-carrying capacity of the bearing and oil film thickness are considered to be a -3 power relationship. Without maximum pump pressure limit, the bearing ability is infinitely large when film thickness approaches zero. In real working conditions, this assumption leads to inaccurate calculations, especially when the oil film is thin. Due to factors such as surface roughness, geometry error, and tilting, a thinner oil film means more chance for film collapse, which seriously affects the service performance of machine tools. Therefore, the oil film of a hydrostatic turntable needs to be regulated to a safe level to avoid damage. The working condition of the hydrostatic turntable can be written as:

$$
\left\{\begin{array}{c}
H \geq H_{0} \\
\sum_{i=1}^{N} W_{i}=m g
\end{array},\right.
$$

where $H_{0}$ is the pre-set film thickness, $W_{i}$ is the loadcarrying capacity of $i^{\text {th }}$ oil pad, $N$ is the total number of oil pads, $m$ is the carrying load, and $g$ is the acceleration of gravity.

\subsection{The Reynolds Equation and FDM Solution}

The Reynolds equation is widely used in the analysis of hydrostatic systems to describe the pressure distribution of viscous oil film in oil pads. The following dimensionless parameters are defined before solving the Reynolds equation:

$$
\bar{p}=\frac{p}{P_{0}}, \quad \overline{p_{0}}=1, \quad \bar{r}=\frac{r}{R_{0}}, \quad \bar{R}_{0}=1, \quad \bar{z}=\frac{z}{H},
$$




$$
\begin{gathered}
\bar{h}=\frac{h}{H}, \quad \bar{H}=1, \quad \bar{U}_{r}=\frac{U_{r}}{\frac{H_{0}^{2} P_{0}}{R_{0} \eta}, \quad \bar{U}_{\theta}=\frac{U_{\theta}}{\frac{H_{0}^{2} P_{0}}{R_{0} \eta}}} \\
\bar{W}=\frac{W}{R_{0}^{2} P_{0}}, \quad \bar{q}=\frac{Q}{\frac{H_{0}^{3} P_{0}}{\eta}},
\end{gathered}
$$

where $p$ is the pressure, $P_{0}$ is the pressure in the oil pocket, $r$ is the radial coordinate, $h$ is the film thickness, $U_{r}$ is the radial velocity, $U_{\theta}$ is the circumferential velocity, $W$ is the load-carrying capacity, $Q$ is the volumetric flow rate of each oil pad, and $\eta$ is the viscosity. Furthermore, $\bar{p}$ is the dimensionless pressure, $\bar{h}$ is the dimensionless film thickness, $\bar{U}_{r}$ is the dimensionless radial velocity, $\bar{U}_{\theta}$ is the dimensionless radial velocity, $\bar{W}$ is the dimensionless load-carrying capacity, and $\bar{q}$ is the dimensionless flow rate.

The oil pocket is usually designed to be of $5 \mathrm{~mm}$ to $10 \mathrm{~mm}$ depth, thicker than the boundary layer of the oil flow. Therefore, no pressure loss exists when the oil flows through the oil pocket. The film thickness at the oil-sealing edge is usually designed to be from tens to hundreds of microns, smaller than the boundary layer of oil flow, so the oil-sealing edge keeps the pressure unchanged in the oil pocket. In a polar coordinate system, the Reynolds equation describing the pressure distribution of the oil film in an oil-sealing edge is written as Eq. (3) [3]:

$$
\begin{gathered}
\frac{\partial}{\partial \bar{r}}\left(\bar{r} \cdot \bar{h}^{3} \cdot \frac{\partial \bar{p}}{\partial \bar{r}}\right)+\frac{\partial}{\partial \bar{\vartheta}}\left(\bar{h}^{3} \cdot \frac{\partial \bar{p}}{\bar{r} \partial \bar{\vartheta}}\right)= \\
\quad=6 \frac{\partial}{\partial \bar{r}}\left(\bar{r} \overline{U_{r}} \bar{h}\right)+6 \frac{\partial}{\partial \bar{\vartheta}}\left(\overline{U_{\vartheta}} \bar{h}\right) .
\end{gathered}
$$

The hydrostatic turntable remains steady in this research, so the velocity can be ignored. The Reynolds equation is modified as Eq. (4):

$$
\frac{\partial}{\partial \bar{r}}\left(\bar{r} \cdot \bar{h}^{3} \cdot \frac{\partial \bar{p}}{\partial \bar{r}}\right)+\frac{\partial}{\partial \bar{\vartheta}}\left(\bar{h}^{3} \cdot \frac{\partial \bar{p}}{\bar{r} \partial \bar{\vartheta}}\right)=0
$$

The discretization of the Reynolds partial differential equation is performed by adopting the FDM method; dimensionless pressure distribution can be determined via the Gauss-Seidel iteration. Therefore, the discrete Reynolds equation is written as [9]:

$$
\left[A 1_{i, j} A 2_{i, j} A 3_{i, j} A 4_{i, j} A 5_{i, j}\right]\left[\begin{array}{c}
\bar{p}_{i+1, j} \\
\bar{p}_{i-1, j} \\
\bar{p}_{i, j} \\
\bar{p}_{i, j-1} \\
\bar{p}_{i, j+1}
\end{array}\right]=0
$$

$$
\left\{\begin{array}{c}
A 1_{i, j}=\frac{\bar{r}_{i} \bar{h}_{i, j}^{3}}{\bar{r}_{\text {step }}^{2}} \\
A 2_{i, j}=\frac{\bar{r}_{i-1} \bar{h}_{i-1, j}^{3}}{\bar{r}_{\text {step }}^{2}} \\
A 3_{i, j}=-\left(\frac{\bar{r}_{i} \bar{h}_{i, j}^{3}}{\bar{r}_{\text {step }}^{2}}+\frac{\bar{r}_{i-1} \bar{h}_{i-1, j}^{3}}{\bar{r}_{\text {step }}^{2}}+\frac{\bar{h}_{i, j-1}^{3}}{\bar{r}_{i} \bar{\theta}_{\text {step }}^{2}}+\frac{\bar{h}_{i, j}^{3}}{\bar{r}_{i} \bar{\theta}_{\text {step }}^{2}}\right) \\
A 4_{i, j}=\frac{\bar{h}_{i, j-1}^{3}}{\bar{r}_{i} \bar{\theta}_{\text {step }}^{2}} \\
A 5_{i, j}=\frac{\bar{h}_{i, j}^{3}}{\bar{r}_{i} \bar{\theta}_{\text {step }}^{2}}
\end{array}\right.
$$

where $\bar{r}_{\text {step }}$ is the step length for the $r$ coordinate, $\bar{\theta}_{\text {step }}$ is the step length for the $\theta$ coordinate, and $i$ and $j$ are numerical counts of the elements on $r$ and $\theta$ coordinate, respectively. The boundary condition of dimensionless pressure $p_{i, j}$ is chosen as 1 in the oil pocket and 0 at the external edge of the oil pad:

$$
\bar{p}_{i, j}=\left\{\begin{array}{ll}
1, & \bar{r}_{i}<\bar{R}_{1} \\
0, & \bar{r}_{i}=1
\end{array} .\right.
$$

The calculation process of FDM will be terminated when the results match the error tolerance $t_{F D M}$. The dimensionless load-carrying capacity of the oil pad can be calculated by integrating the hydrostatic dimensionless pressure distribution, while the dimensionless flow rate is determined by integrating the outer flow speed on the external surface. The load-carrying performance is written as Eq. (8):

$$
\left\{\begin{array}{c}
\bar{w}=\bar{p} \bar{r} d \bar{r} d \bar{\theta} \\
\bar{q}=\int \frac{\bar{z}^{2}-\bar{z}}{2 \eta} \frac{\partial \bar{p}}{\partial \bar{r}} d \bar{z}, \quad \text { where } \bar{r}_{i}=1
\end{array}\right.
$$

$\bar{w}$ and $\bar{q}$ are important parameters to evaluate the supporting performance of hydrostatic bearings, which varies with the changing of $\bar{R}_{1}$ value. For the safe operation of the machine tool, a minimum film thickness $H_{0}$ is chosen to avoid damaging the machine tools. The safety film thickness is set as $H \geq H_{0}$. Assuming the carrying weight is constant, the pump power $P_{s}$ is [10]:

$$
\left\{\begin{array}{l}
P_{0}=\frac{m g}{N \bar{w} \pi R_{0}^{2}} \\
Q=\bar{q} \frac{H_{0}^{3} P_{0}}{\eta} \\
P_{s}=N \cdot P_{0} \cdot Q
\end{array}\right.
$$


where $P_{s}$ is the supporting power consumption, and it only describes the pump power caused in oil pads. To control variables in this optimization, motor efficiency and pressure losses are ignored.

\subsection{Particle Swarm Optimization}

PSO is an emerging optimization method with high computational and implementation efficiency. The analysis of the hydrostatic turntable using the FDM technique requires performing many numerical calculations that make the process very timeconsuming. Different values of $R_{1}$ result in different $\bar{w}$ and $\bar{q}$, i.e. different supporting power consumption. Aiming at the pump power optimization, many calculation loops are required to calculate the optimal value of $R_{1}$, so PSO is a suitable method to efficiently reduce the calculation time and simplify the optimization process. In this paper, PSO is basically performed by evaluating the fitness of each individual in the domain to search for a fitting range until it matches error tolerance. An inertia weight $\omega$ is introduced to improve local search ability [16]:

$$
\left\{\begin{array}{c}
v_{n}^{(k+1)}=\omega v_{n}^{(k)}+c_{1}\left(\text { Pbest }_{n}-x_{n}^{(k)}\right)+c_{2}\left(\text { Gbest }_{n}-x_{n}^{(k)}\right), \\
x_{n}^{(k+1)}=x_{n}^{(k)}+v_{n}^{(k+1)}
\end{array}\right.
$$

where $x_{n}$ and $v_{n}$ are the position and velocity of the $n^{\text {th }}$ individual, respectively, Pbest ${ }_{n}$ is the position of the privative best fitness of the $n^{\text {th }}$ individual, Gbest $t_{n}$ is the position of the global best fitness of all individuals, and $k$ is the loop iteration count.

Every individual represents a value of $R_{1}$, and makes $R_{1}=x_{n} \cdot \mathrm{R}_{0}$. FDM can solve and case by case. The fitness of each individual is evaluated by the supporting power consumption. The minimization of pump power is chosen as the optimal target. Computing will be terminated when every single individual as while as the whole swarm matches the error tolerance. The design tolerance of oil pad is generally at the millimetre scale, so error tolerance of PSO is set as $10^{-2}$ [13]. The terminating condition is:

$$
\left\{\begin{array}{c}
\left|x_{n}^{(k)}-x_{n}^{(k-1)}\right| \leq \text { tol }_{P S O} \\
\max \left(x_{n}^{(k)}\right)-\min \left(x_{n}^{(k)}\right) \leq \text { tol }_{P S O}
\end{array} .\right.
$$

The algorithm presented in this work is mainly composed of three parts: FDM solution of the Reynolds equation, evaluation of supporting power and PSO for minimal power consumption; Fig. 3 illustrates the detailed calculation process [12].

\section{NUMERICAL RESULTS AND DISCUSSION}

\subsection{Influence of Dimensionless Pocket Scale on the Load- Carrying Performance of Circular Oil Pads}

The optimal pocket size can be determined by the algorithm shown in Fig. 3 for minimum supporting power consumption. Table 1 lists the values of the

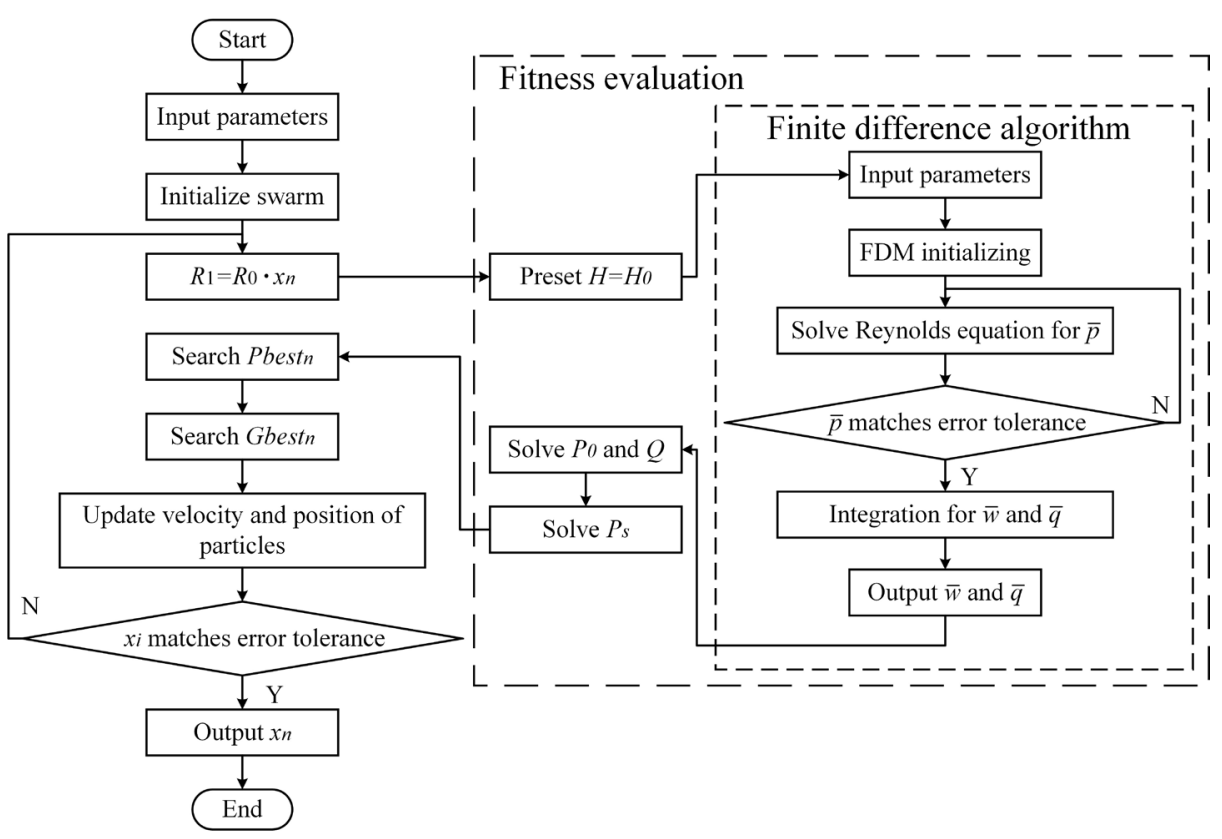

Fig. 3. The PSO implementation framework 
main parameters involved in the computational process.

Table 1. Values/Ranges of major parameters

\begin{tabular}{cccc}
\hline Parameter & Value & Parameter & Value \\
\hline$R_{0}$ & $0.085 \mathrm{~m}$ & $n$ & 5 \\
\hline$m$ & $24800 \mathrm{~kg}$ & $\omega$ & 0.2 \\
\hline$N$ & 20 & $c_{1}$ & 0.4 \\
\hline$\eta$ & $0.06 \mathrm{~Pa} \cdot \mathrm{s}$ & $c_{2}$ & 0.9 \\
\hline$T o l_{F D M}$ & $1 \times 10-7$ & $T o l_{P S O}$ & 6 \\
\hline
\end{tabular}

The supporting performance of an oil pad can be described by dimensionless load-carrying capacity and dimensionless flow rate. The dimensionless loadcarrying capacity corresponds to the force output of oil pads, while the dimensionless flow rate corresponds to the oil flow required to maintain the film thickness. Changes in oil pocket size will influence both the dimensionless carrying capacity and dimensionless flow rate. The pressure distribution for different oil pocket sizes is shown in Fig. 4.

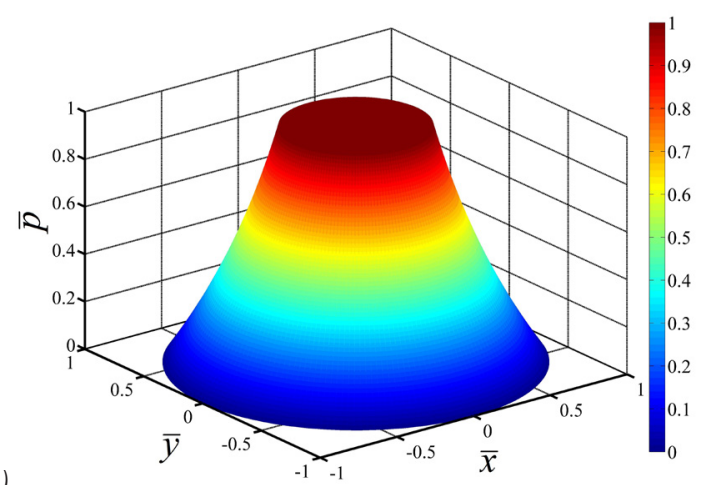

As shown in Fig. 4, the oil pocket is filled with pressurized oil which provides most of the loadcarrying capacity of the oil pad. Therefore, a larger oil pocket means greater load-carrying force. With dimensionless oil pocket size increases, the slope at the oil film sealing edge grows leading to the increase of the dimensionless flow rate. Fig. 5 shows the dimensionless pocket size effect on the supporting performance of the oil pad.

From Fig. 5, both the dimensionless carrying capacity $\bar{w}$, and flow rate $\bar{q}$ increase with the increase of pocket size $\bar{R}_{1}$. Flow rate $\bar{q}$ grows more significantly than $\bar{w}$. A larger dimension of the pocket means greater supply flow rate and lower pump pressure. The supporting power consumption is highly related to the supply flow rate and pump pressure. The difference between the influence of the pocket dimension on the supply flow rate and the pump pressure shows that there is room for improvement. When $\bar{R}_{1}$ varies within the range $(0.1,0.9)$, the influence of the pocket on supporting power is shown a)

Fig. 4. Dimensionless pressure distribution of ci

b)

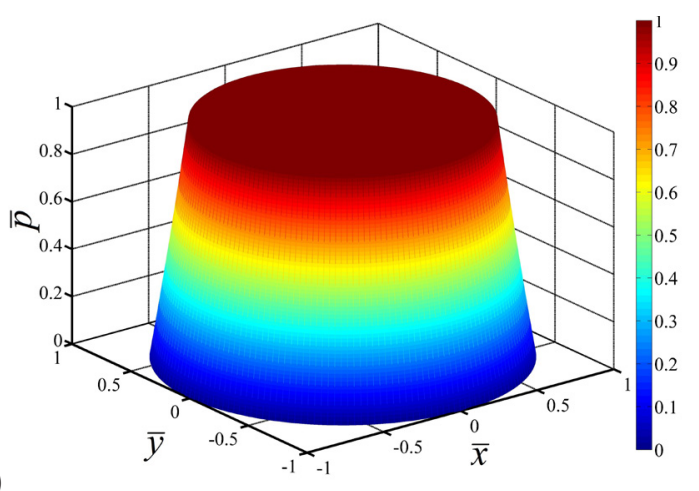

cular oil pad; a) $\bar{R}_{1}=0.4$, and b) $\bar{R}_{1}=0.8$

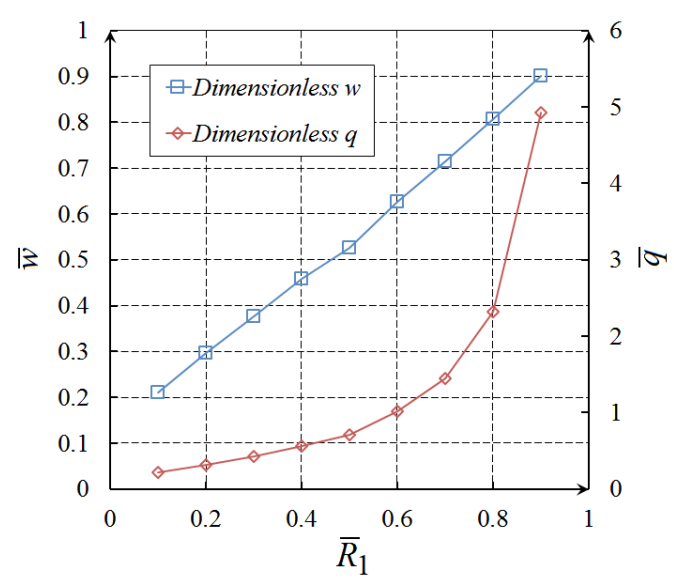

Fig. 5. Supporting performance of circular oil pad for different $\bar{R}_{1}$; a) dimensionless load-carrying capacity and flow rate, and $b)$ pressure and oil supply rate 
in Fig. 6, and a minimum value of the curve of supporting power $P_{s}$ clearly exists:

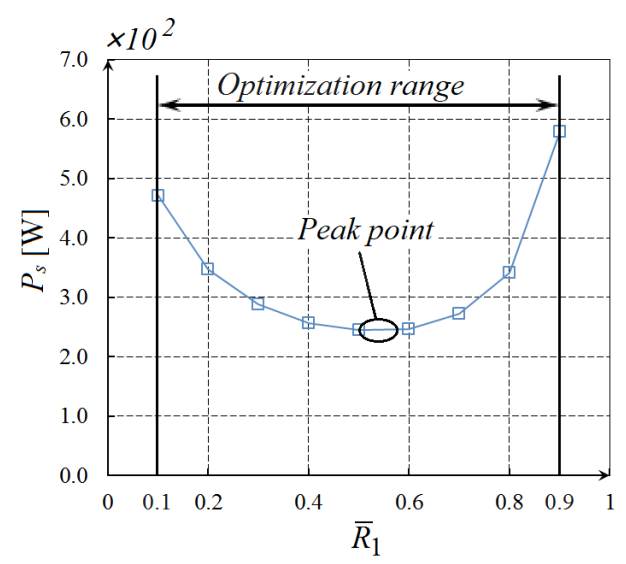

Fig. 6. Supporting power consumption varying $\bar{R}_{1}$

However, the exhaustive method can only show the trend vaguely and thus is not suitable for solving optimal result. The exact place where $P_{s}$ achieves its minimum needs to be determined with the help of PSO.

\subsection{Experimental Verification on a Miniature Oil Pad}

Due to cost and safety constraints, it is difficult to carry out experiments on a hydrostatic turntable. To verify the theoretical calculations, an experiment table for a single oil pad is designed. The carrying performance of a replaceable oil pad can be measured under different bearing situations. Oil is supplied by a pump, and the flow rate is adjusted by a flow valve. Film thickness can be changed by a lead screw with a very large reduction ratio. The film thickness varies $2 \mu \mathrm{m}$ for every turn of the screw hand wheel. Three force sensors, two dial indicators, one flowmeter, one manometer, and one thermometer are deployed to measure related parameters. Data from each sensor is stored by a collector by real time. The experimental table is shown in Fig. 7.

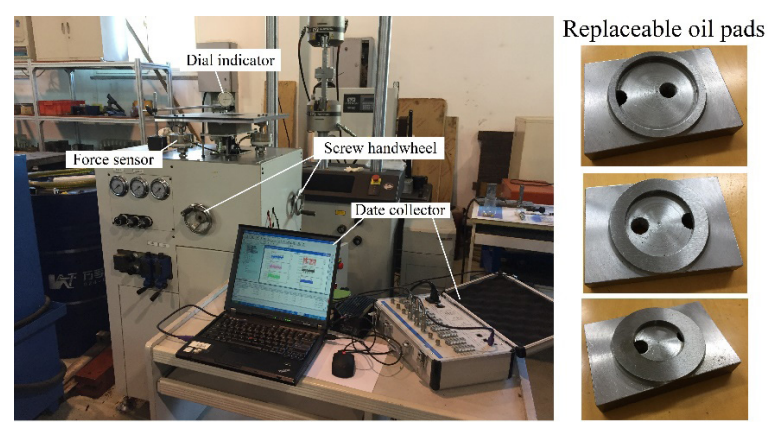

Fig. 7. Experimental table to test single oil pad

To verify the reliability of the theoretical algorithm, four different oil pads are tested. Their structural parameters are shown in Table 2.

Table 2. Structural parameters of testing oil pads

\begin{tabular}{lcll}
\hline & $R_{0}$ & \multicolumn{1}{c}{$R_{1}$} & \multicolumn{1}{c}{$\bar{R}_{1}$} \\
\hline $\operatorname{Pad} 1$ & $0.033 \mathrm{~m}$ & $0.028 \mathrm{~m}$ & 0.848485 \\
$\operatorname{Pad} 2$ & $0.033 \mathrm{~m}$ & $0.023 \mathrm{~m}$ & 0.69697 \\
$\operatorname{Pad} 3$ & $0.033 \mathrm{~m}$ & $0.018 \mathrm{~m}$ & 0.545455 \\
$\operatorname{Pad} 4$ & $0.033 \mathrm{~m}$ & $0.013 \mathrm{~m}$ & 0.393939 \\
\hline
\end{tabular}

Several tests are conducted to determine the dimensionless carrying ability and flow rate of oil pads, based on Eq. (2). Compared with the theoretical result, the experimental results of dimensionless carrying ability are smaller, and the maximum error is about $18 \%$. Deviation also appears between the
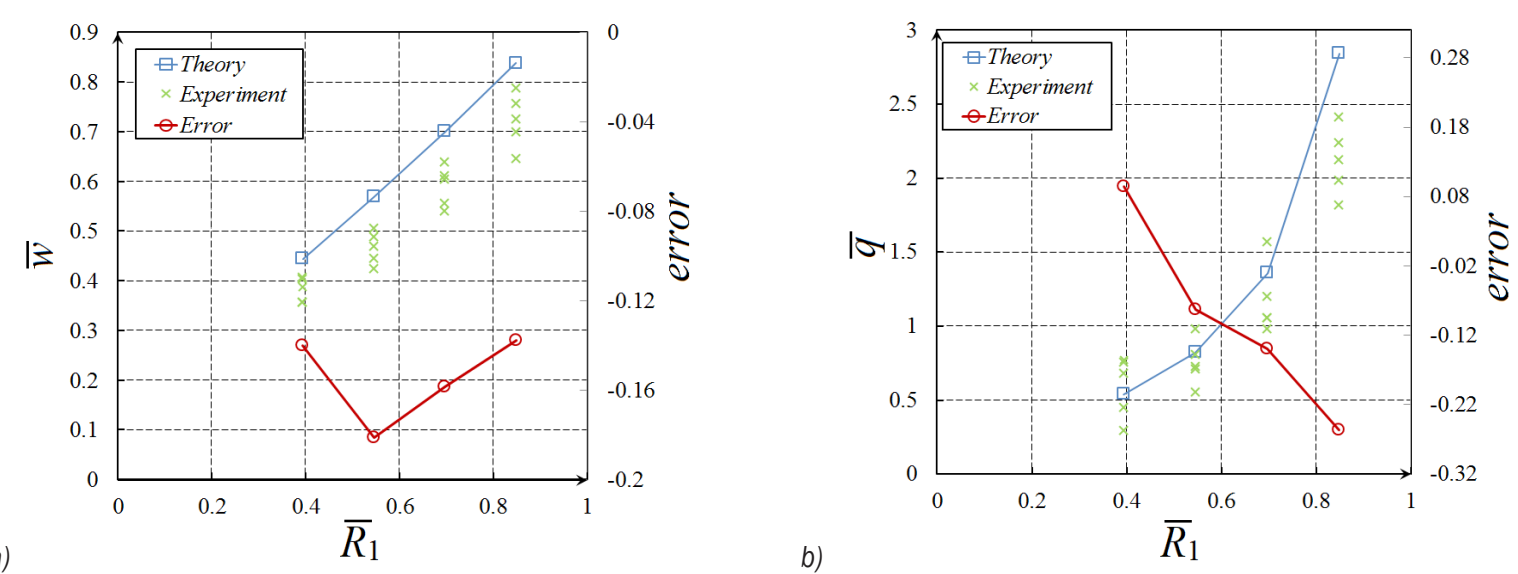

Fig. 8. Comparison between theoretical and experimental results; a) dimensionless carrying force, b) dimensionless flow rate 




Fig. 9. Power estimate of single oil pad testing

theoretical and experimental dimensionless flow rates. The maximum error is about $25 \%$. Although there are some errors between the calculation and tests, the varying trend of dimensionless carrying force and flow rate with $\bar{R}_{1}$ is the same as predicted.

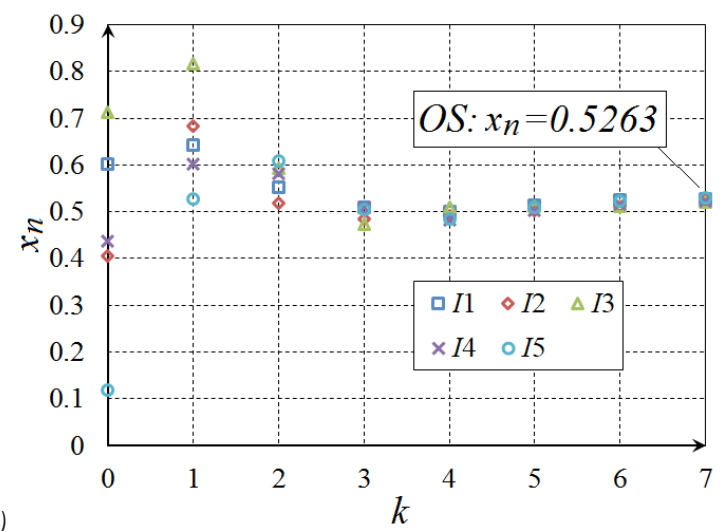

According to the result shown in Fig. 6, there is an optimal value of $\bar{R}_{1}$ to achieve minimum supporting power $P_{s}$. The supporting power is calculated based on the experimental result shown in Fig. 8.

As shown in Fig. 9, both the theoretical and experimental result shows a minimum $P_{s}$ exists. The following optimization is carried out by the real hydrostatic turntable parameters shown in Table 1.

\subsection{Optimal Solution of Oil Pocket Scale Based on Particle Swarm Optimization}

The initial position and speed of particles $x_{n}$ are chosen randomly in the domain. Assuming $R_{1}=x_{n} \cdot \mathrm{R}_{0}$, the oil pocket scale is related with particle positions. The film thickness is set to $H_{0}$, and the supporting performance is solved by FDM to determine the pump power consumption. PSO can determine the position of minimum power consumption by evaluating the fitness of each particle. Fig. 10 shows the iteration process of PSO when $H_{0}=5 \times 10^{-5} \mathrm{~m}$, where $O S$ is optimal solution determined by PSO and $I 1, I 2, \ldots$,

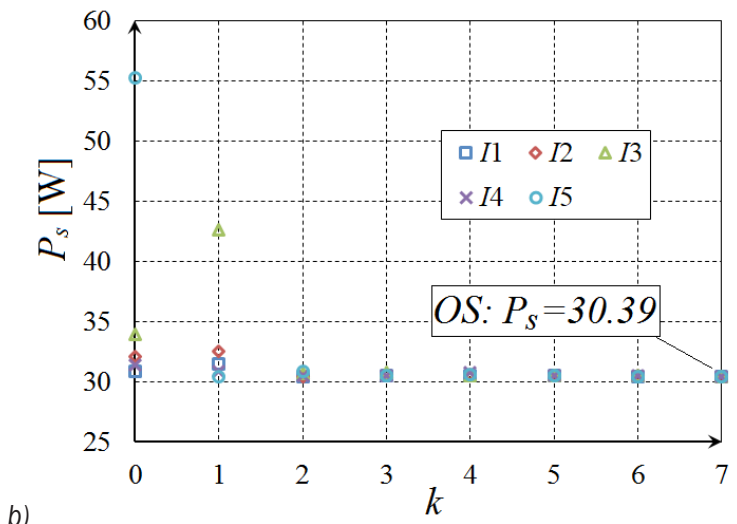

Fig. 10. Iterative process of PSO $\left(H_{0}=5 \times 10^{-5} \mathrm{~m}\right)$; a) position of each individual, b) supporting power of each individual


Fig. 11. Iterative process of PSO $\left(H_{0}=10 \times 10^{-5} \mathrm{~m}\right)$; a) position of each individual, and b) supporting power of each individual 


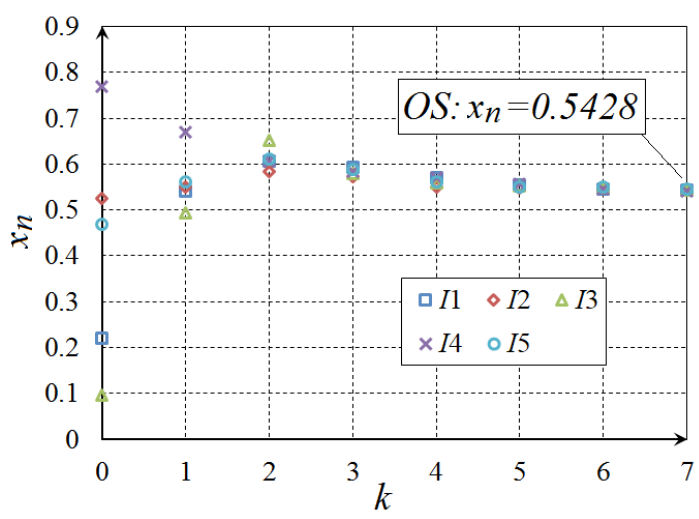

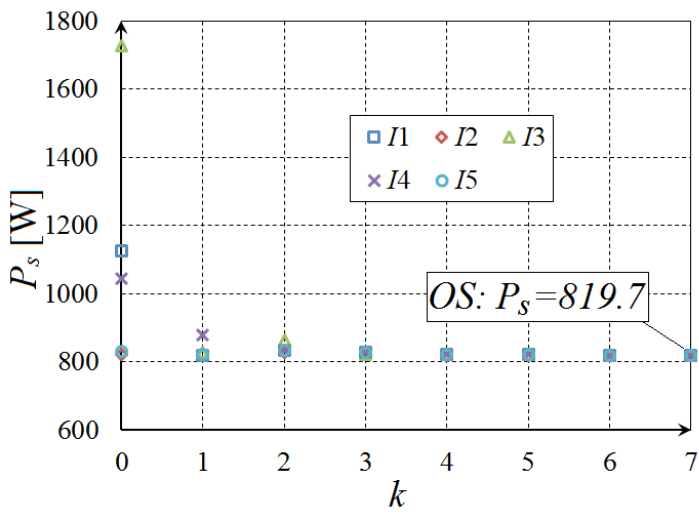

b)

Fig. 12. Iterative process of PSO $\left(H_{0}=15 \times 10^{-5} \mathrm{~m}\right)$; a) position of each individual, and b) supporting power of each individual

In are individuals. From Fig. 7, the algorithm needs 7 loops to meet the error tolerance when the number of particle swarms is chosen as 5. Individuals get close to each other rapidly from distinct positions and converge to $x_{n}=0.5263$ where $P_{s}=30.39$. Figs. 11 and 12 show the iteration process of PSO when $H_{0}$ is set to $10 \times 10^{-5} \mathrm{~m}$ and $15 \times 10^{-5} \mathrm{~m}$, respectively.

The algorithm needs 7 loops to meet the error tolerance. When $H_{0}=10 \times 10^{-5} \mathrm{~m}$, all particles converge to $x_{n}=0.5467$ where $P_{s}=242.9$. When $H_{0}=$ $15 \times 10^{-5} \mathrm{~m}$, all particles converge to $x_{n}=0.5428$ where $P_{s}=819.7$. The change of $H_{0}$ value influences the final result $x_{n}$ by no more than $3.9 \%$. However, varying $H_{0}$ has a strong effect on $P_{s}$, meaning a thicker film thickness needs a higher supporting power. Taking 14 different samples of $H_{0}$, the relationship between film thickness, pump power consumption, and optimal pocket size is shown in Fig. 13.

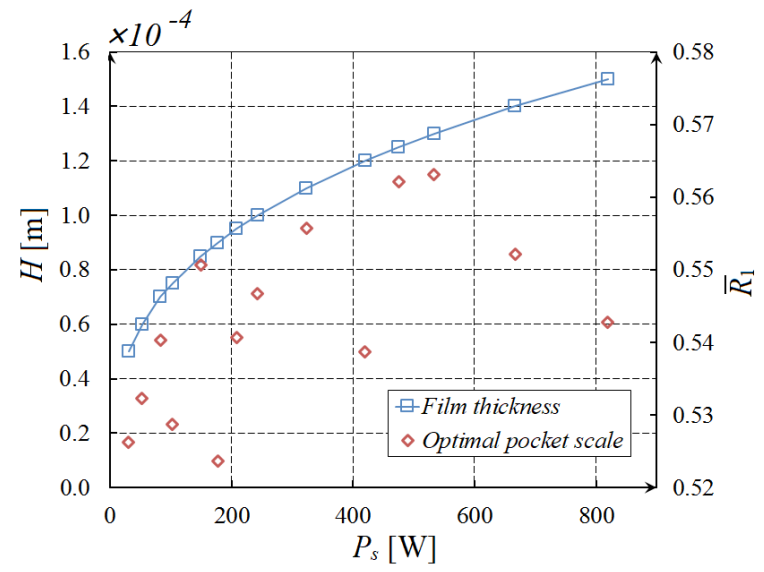

Fig. 13. Relationship between film thickness, supporting power and oil pocket scale
The supporting power consumption raises with the film thickness increase. The optimal pocket scale changes no more than $7 \%$ under different film thickness, a percentage that could be considered as a computational error. The average optimal pocket size of 14 samples is determined as $\bar{R}_{1}=0.5431$.

\section{CONCLUSIONS}

In this work, the load-carrying performance of a hydrostatic turntable is analysed using an FDM solution of the Reynolds equation. The relationship between oil pocket scale and supporting power consumption is determined numerically. The reliability of the algorithm is verified by conducting experiments on a miniature testing table. PSO is carried out to solve the optimal pocket size for minimal pump power. An efficient and practical optimization method for hydrostatic turntable design is presented, and the following conclusions can be drawn:

1. The oil pocket scale strongly influences the supporting power consumption. A properly designed oil pocket reduces the supporting power and improves the service performance of the hydrostatic turntable.

2. When the film thickness is set as a constant (to a safety value), the optimal pocket size of $\bar{R}_{1}$ $=0.5431$ is calculated. With increases in film thickness, the supporting power consumption grows, but optimal pocket size remains nearly the same. The optimal oil pocket scale varies no more than $7 \%$ under different film thicknesses.

3. The design tolerances of the oil pad are at a millimetre scale, and the FDM analysis processes are always very time-consuming. Therefore, the PSO is a suitable optimization method to speed up calculations and overcome such issues. 


\section{ACKNOWLEDGMENT}

The authors would like to thank the National Natural Science Fund coded 51575009, the National Science and Technology Major Project coded 2018ZX04043, Beijing Natural Science Fund coded 3162003 and Jing-Hua Talents Project of Beijing University of Technology for supporting the research.

\section{NOMENCLATURES}

\begin{tabular}{|c|c|}
\hline$A 1_{i, j}, A 2$ & coefficient matrix, [-] \\
\hline$g$ & acceleration of gravity, $\left[\mathrm{m} \cdot \mathrm{s}^{-2}\right]$ \\
\hline Gbest $_{n}$ & $\begin{array}{l}\text { position of the global best fitness of all } \\
\text { individuals, [-] }\end{array}$ \\
\hline$H$ & film thickness, $[\mathrm{m}]$ \\
\hline$H_{0}$ & preset film thickness, $[\mathrm{m}]$ \\
\hline $\bar{h}$ & dimensionless film thickness, [-] \\
\hline$i$ & $\begin{array}{l}\text { numerical counts of the elements on } r \\
\text { coordinate }\end{array}$ \\
\hline j & $\begin{array}{l}\text { numerical counts of the elements on } \theta \\
\text { coordinate, [-] }\end{array}$ \\
\hline$k$ & loop iteration count, [-] \\
\hline$N$ & total number of oil pads, [-] \\
\hline$m$ & carrying load, $[\mathrm{kg}]$ \\
\hline$p$ & pressure, $[\mathrm{Pa}]$ \\
\hline$P_{0}$ & pressure in the oil pocket, $[\mathrm{Pa}]$ \\
\hline$P_{S}$ & supporting power, [W] \\
\hline $\bar{p}$ & dimensionless pressure, $[-]$ \\
\hline Pbest $_{n}$ & $\begin{array}{l}\text { position of the privative best fitness } \\
\text { of the } n^{\text {th }} \text { individual, [-] }\end{array}$ \\
\hline$R_{0}$ & radius of oil pad, $[\mathrm{m}]$ \\
\hline$R_{1}$ & radius of oil pocket, $[\mathrm{m}]$ \\
\hline $\bar{r}_{\text {step }}$ & step length for the $r$ coordinate, $[-]$ \\
\hline$Q$ & volumetric flow rate, $\left[\mathrm{m}^{3} \cdot \mathrm{s}^{-1}\right]$ \\
\hline $\bar{q}$ & dimensionless flow rate, [-] \\
\hline$U_{r}$ & radial velocity, $\left[\mathrm{m} \cdot \mathrm{s}^{-1}\right]$ \\
\hline$U_{\theta}$ & circumferential velocity, $\left[\mathrm{m} \cdot \mathrm{s}^{-1}\right]$ \\
\hline $\bar{U}_{r}$ & dimensionless radial velocity, [-] \\
\hline $\bar{U}_{\theta}$ & dimensionless radial velocity, [-] \\
\hline$v_{n}$ & velocity of the $n^{\text {th }}$ individual, [-] \\
\hline$x_{n}$ & position of the $n^{\text {th }}$ individual, [-] \\
\hline$W$ & load-carrying capacity, $[\mathrm{N}]$ \\
\hline$W_{i}$, & load-carrying capacity of $i^{\text {th }}$ oil pad, $[\mathrm{N}]$ \\
\hline $\bar{W}$ & dimensionless carrying capacity, [-] \\
\hline$\eta$ & viscosity, $[\mathrm{Pa} \cdot \mathrm{s}]$ \\
\hline $\bar{\theta}_{\text {step }}$ & step length for the $\theta$ coordinate \\
\hline
\end{tabular}

\section{REFERENCES}

[4] Wang, N., Cha, K.-C., Huang, H.-C. (2012). Fast convergence of iterative computation for incompressible-fluid Reynolds equation. Journal of Tribology, vol. 134, no. 4, p. 024504 , DOl:10.1115/1.4006360.

[5] Singh, U.P., Gupta, R.S., Kapur, V.K. (2012). On the steady performance of annular hydrostatic thrust bearing: Rabinowitsch fluid model. Journal of Tribology, vol. 134, no. 4, p. 044502, DOI:10.1115/1.4007350.

[6] Liu, Z., Wang, Y., Cai, L., Cheng, Q., Zhang, H. (2016). Design and manufacturing model of customized hydrostatic bearing system based on cloud and big data technology. The International Journal of Advanced Manufacturing Technology, vol. 84, no. 1-4, p. 261-273, D0l:10.1007/s00170-015-8066-2.

[7] El Khlifi, M., Souchet, D., Hajjam, M., Bouyahia, F. (2007). Numerical modeling of non-Newtonian fluids in slider bearings and channel thermohydrodynamic flow. Journal of Tribology, vol. 129, no. 3, p. 695-699, D0l:10.1115/1.2736732.

[8] Mankovits, T., Szabó, T., Kocsis, I., Páczelt, I. (2014). Optimization of the shape of axi-symmetric rubber bumpers. Strojniški vestnik - Journal of Mechanical Engineering, vol. 60, no. 1, p. 61-67, D0l:10.5545/sv-jme.2013.1315.

[9] Gustafsson, T., Rajagopal, K.R., Stenberg, R., Videman, J. (2015). Nonlinear Reynolds equation for hydrodynamic lubrication. Applied Mathematical Modelling, vol. 39, no. 17, p. 5299-5309, DOl:10.1016/J.apm.2015.03.028.

[10] Yang, Q., Huang, P., Fang, Y. (2016). A novel Reynolds equation of non-Newtonian fluid for lubrication simulation. Tribology International, vol. 94, p. 458-463, D0l:10.1016/j. triboint.2015.10.011.

[11] Masjedi, M, Khonsari, M.M. (2015). On the effect of surface roughness in point-contact EHL: Formulas for film thickness and asperity load. Tribology International, vol. 82, part A, p. 228-244, D0I:10.1016/j.triboint.2014.09.010.

[12] Deresse, G.A., Sinha, P. (2011). THD analysis for finite slider bearing with roughness: special reference to load generation in parallel sliders. Acta Mechanica, vol. 222, no. 1-2, p. 1-15, D0l:10.1007/s00707-011-0515-x.

[13] Cai, L., Wang, Y., Liu, Z., Cheng, Q. (2015). Carrying capacity analysis and optimizing of hydrostatic slider bearings under inertial force and vibration impact using finite difference method (FDM). Journal of Vibroengineering, vol. 17, no. 6, p. 2781-2794.

[14] Weißbacher, C., Schellnegger, C:, John, A., Buchgraber, T., Pscheidt, W. (2014). Optimization of journal bearing profiles with respect to stiffness and load-carrying capacity. Journal of Tribology, vol. 136, no. 3, p. 031709, D0l:10.1115/1.4027399.

[15] Cheng, Q., Zhan, C., Liu, Z., Zhao, Y., Gu, P. (2015). Sensitivitybased multidisciplinary optimal design of a hydrostatic rotary table with particle swarm optimization. Strojniški vestnik Journal of Mechanical Engineering, vol. 61, no. 7-8, p. 432447, DOI:10.5545/sv-jme.2015.2478.

[16] Chang, S.H., Jeng, Y.R. (2013). A modified particle swarm optimization algorithm for the design of a doublepad aerostatic bearing with a pocketed orifice-type restrictor. Journal of Tribology, vol. 136, no. 2, p. 021701 , DOI:10.1115/1.4026061. 
[17] Chan, C.-W. (2015). Modified particle swarm optimization algorithm for multi-objective optimization design of hybrid journal bearings. Journal of Tribology, vol. 137, no. 2, p. 021101, D0I:10.1115/1.4028606.

[18] Soltani-Mohammadi, S., Safa, M., Mokhtari, H. (2016). Comparison of particle swarm optimization and simulated annealing for locating additional boreholes considering combined variance minimization. Computers \& Geosciences, vol. 95, p. 146-155, D0l:10.1016/j.cage0.2016.07.020.

[19] Zheng, Y.-X., Liao, Y. (2016). Parameter identification of nonlinear dynamic systems using an improved particle swarm optimization. Optik - International Journal for Light and Electron Optics, vol. 127, no. 19, p. 7865-7874, Dol:10.1016/j.jileo.2016.05.145.

[20] Srisha Rao, M.V., Jagadeesh, G. (2010). Vector evaluated particle swarm optimization (VEPSO) of supersonic ejector for hydrogen fuel cells. Journal of Fuel Cell Science and Technology, vol. 7, no. 4, p. 041014, D0l:10.1115/1.4000676.
[21] Flocker, F.W., Bravo, R.H. (2016). On global convergence in design optimization using the particle swarm optimization technique. Journal of Mechanical Design, vol. 138, no. 8, p. 081402, D0I:10.1115/1.4033727.

[22] Tang, D., Dai, M., Salido, M.A., Giret, A. (2016). Energyefficient dynamic scheduling for a flexible flow shop using an improved particle swarm optimization. Computers in Industry, vol. 81, p. 82-95, D0l:10.1016/j. compind.2015.10.001.

[23] Luo, W., Sun, J., Bu, C., Liang, H. (2016). Species-based particle swarm optimizer enhanced by memory for dynamic optimization. Applied Soft Computing, vol. 47, p. 130-140, D0l:10.1016/j.asoc.2016.05.032.

[24] Liu, Z., Wang, Y., Cai, L., Zhao. Y., Cheng, Q., Dong, X. (2017). A review of hydrostatic bearing system: Researches and applications. Advances in Mechanical Engineering, vol. 9, no. 10, p. 1-27, DOI:10.1177/1687814017730536. 